\title{
La construcción en el Producto Interno Bruto del Ecuador, 2000-2018
}

\section{Construction in the Gross Domestic Product of Ecuador, 2000-2018}

INFORMACIÓN DEL

\section{ARTÍCULO}

Fecha de recepción: 22 de Octubre de 2018.

Fecha de aceptación: 6 de Mayo de 2019

${ }^{I}$ Magíster en Relaciones Internacionales, Universidad Andina Simón Bolívar. Docente e Investigador de la Universidad UTE-Ecuador.

E-mail: nelsone.garcia@ute.edu.ec.

Código ORCID

https://orcid.org/0000-0002-2286-3793

2 Magíster en Educación Superior, Universidad Central del Ecuador. Docente e Investigadora de la Universidad UTE-Ecuador.

E-mail: ximena.tobar@ute.edu.ec.

Código ORCID:

https://orcid.org/0000-0003-0898-7025

CITACIÓN: García Osorio, N. E., \& Tobar Cazares, X. del C. (2019). La construcción en el Producto Interno Bruto del Ecuador, 2000-2018. Podium, $35,57-68$

doi:10.31095/podium.2019.35.4

ENLACE DOI:

http://dx.doi.org/10.31095/podium.201 9.35 .4
Nelson Euclides García Osorio ${ }^{1}$, Ximena del Consuelo Tobar Cazares ${ }^{2}$

\section{Resumen}

Este artículo tiene por objeto examinar la influencia del sector de la construcción en el comportamiento económico del Ecuador. Se investigaron las relaciones entre el sector de la construcción y el Producto Interno Bruto (PIB) nacional, sus variaciones durante el periodo 2000 - 2018, y los cambios exógenos en el mencionado sector. Se recopiló información cuantitativa y descriptiva de las publicaciones estadísticas oficiales relacionadas con el PIB, la construcción, y la población ocupada, confirmándose que la industria de la construcción es sensible a la dinámica de la economía nacional, más aún, por la política económica de los gobiernos que no se ubicó claramente en el estatismo o capitalismo.

\section{Palabras Clave:}

Construcción, variación, participación, Producto Interno Bruto, comportamiento económico, ciclos económicos.

Clasificación JEL: E02, E12.

\begin{abstract}
The purpose of this article is to examine the influence of the construction sector on the economic behavior of Ecuador. The relationships between the construction sector and the national Gross Domestic Product (GDP), its variations during the period $2000-2018$, and the exogenous changes in the aforementioned sector were investigated. Quantitative and descriptive information was collected from the official statistical publications related to GDP, construction, and the employed population, confirming that the construction industry is sensitive to the dynamics of the national economy, moreover, due to the economic policy of the governments that were not clearly placed in statism or capitalism.
\end{abstract}

\section{Keywords:}

Construction, variation, participation, Gross Domestic Product, economic behavior, economic cycles.

JEL Classification: E02, E12. 


\section{Introducción}

Los ingresos que financian el Presupuesto General del Estado son: petroleros, no petroleros ${ }^{3}$, y del resultado operacional de las empresas públicas. La evolución de los sectores de la economía obedece a la implementación de la política económica de los gobiernos de turno del país, cabe señalar que el gobierno que más tiempo estuvo en el poder, desde el regreso al régimen democrático, es el de Rafael Correa quien implementó una política de Estado intervencionista, aumentando progresivamente sus gastos y multiplicando las responsabilidades, minimizando al sector privado y estableciendo el proteccionismo condicionado por sus convicciones socialistas (Hurtado, 2017), sin embargo, por la escasez de recursos fiscales Correa cambió al modelo neokeynesiano. Estefanía (2016) deduce, si el liberalismo económico basaba su pensamiento en la preeminencia del mercado y el marxismo en la planificación central y en la potenciación del estado, el keynesianismo adoptó una sabia mezcla de ambos como método de corrección de los problemas económicos.

El propósito de este estudio es demostrar el grado de influencia del sector de la construcción en el comportamiento económico del Ecuador mediante el análisis del Producto Interno Bruto (PIB) Nacional, determinando los ciclos económicos en el periodo 2000 2018. A lo largo de la historia el sector

\footnotetext{
${ }^{3}$ Los ingresos no petroleros lo conforman: Ingresos tributarios (impuesto a la renta, IVA, ICE, arancelarios y otros impuestos), contribuciones a la seguridad social y otros.
}

que más ha contribuido al PIB Nacional es el de la manufactura (excepto refinación de petróleo). La construcción tiene una participación importante no sólo por su encadenamiento productivo, sino también porque emplea mano de obra no calificada y calificada vinculada al sector inmobiliario, de tal manera, que contribuyó en promedio con el $8.4 \%$ de la producción total de la economía nacional durante el mencionado periodo (Banco Central del Ecuador, 2018).

Existen investigaciones relacionadas con el sector de la construcción y las crisis económicas en donde se resalta "la alta incidencia macroeconómica del sector inmobiliario y su rol articulador entre el sector financiero y la economía real", (Daher, 2013, p.1). En el caso ecuatoriano esta industria depende de las políticas macroeconómicas establecidas por los gobiernos, reflejados en la variación del sector real de la economía. Velasco (2015) refiere un informe de Hermel Flores, ex presidente de la Cámara de la Industria de la Construcción, donde se afirma que después de un crecimiento significativo de la economía existió una desaceleración en el sector de la construcción debido principalmente a la reducción de la inversión pública. En todo caso, las acciones de capital público (edificios e infraestructura) junto con acciones de capital privado, constituyen el stock de capital total. Sin embargo, Ahmadi y Shahandashti (2017) manifiestan que la base para la inclusión del capital social total es que independientemente del tipo de propiedad (pública o privada), cuanto mayor sea éste, menor será el efecto del 
crecimiento del sector de la construcción en el crecimiento económico y viceversa.

\section{Revisión de literatura}

Antes de la última crisis del 2008, las instituciones financieras $\mathrm{y}$ fondos de inversión inmobiliaria transnacionales encontraron en la financiarización y titulación una vía eficaz para su diversificación geográfica y distribución del riesgo hipotecario (Daher, 2013). En Ecuador después de la crisis financiera de 1998, la Industria de la Construcción depende de las políticas macroeconómicas establecidas por los gobiernos, reflejadas en la variación del sector real de la economía. Kumar (2011), afirma que la expansión de la actividad de la construcción está encabezada por un aumento en la expansión económica. En el año 2013 la Industria de la Construcción alcanzó su peak, representando un $10.46 \%$ del PIB Nacional, debido a los ingresos provenientes del aumento de los precios del petróleo, según datos del Ministerio de Finanzas (Durán, 2017). En el sector real existieron variaciones significativas. Rincón y Torres (2013) al respecto exponen que la escuela neokeynesiana restaura el debate científico en los temas macroeconómicos de mayor relevancia como son: el crecimiento, los ciclos económicos, salarios eficientes, la regulación de los mercados, política monetaria y política fiscal.

La estructura porcentual de la Formación Bruta de Capital Fijo (inversión) del sector público en los periodos 2000 - 2006 y $2007-2016$ en promedio fue de 27.2 y 45.6, respectivamente. Mientras que en el sector privado en los mismos periodos pasó del 72.8 al 54.4 (Banco Central del Ecuador, 2018). Se evidenció un incremento de la inversión pública en 18.4 puntos porcentuales. Para los post keynesianos el gasto es el responsable de determinar el ingreso y el empleo. En este sentido, un incremento de la demanda agregada, generada por un incremento en el gasto público podría aumentar el empleo a través de un ajuste en la producción (Radziunas, 2005).

Los neokeynesianos nacen en un entorno de declinación del monetarismo y ocaso del neoliberalismo (1980-2000), con una teoría del crecimiento avanzado con poblaciones de alta educación (Giudice, 2004). Rincón y Torres (2013) afirman que el Keynesianismo fue adoptado como un paradigma dominante en todas las universidades occidentales, liderada por las propuestas de Samuelson, quien realiza la síntesis teórica entre las diversas corrientes dando lugar al neokeynesianismo como una fusión de la teoría neoclásica con la keynesiana. La política económica consiste en las medidas que adopta el gobierno enmarcado en un modelo económico, a través de la política fiscal y de la política monetaria, para corregir problemas de liquidez que se reflejan por la desaceleración económica (Sachs y Larraín, 1994), como sucedió en la última crisis mundial originada en New York, cuando los bancos de Wall Street intentaron desprenderse de sus Collateralize Debt Obligations (CDO), se dieron cuenta que no existía un mercado para ellos. Las compañías de seguros que 
habían asegurado los CDO con CDS comprobaron que su mercado también colapsaba (Gowan, 2009).

\section{Metodología}

La investigación es de carácter cuantitativa y descriptiva. Se recopiló la información a partir de las publicaciones estadísticas relacionadas con las industrias o sectores económicos que conforman el Producto Interno Bruto del Ecuador, así como también indicadores de la población empleada, que se encuentran disponibles en el Banco Central del Ecuador en su boletín de cuentas nacionales, y en el Instituto Nacional de Estadísticas y Censos (INEC). Los datos numéricos fueron procesados para el cálculo de las tasas de variación anual, promedio y de participación para analizar la evolución y comportamiento de las mencionadas variables y determinar los ciclos económicos y su influencia en el PIB Construcción durante el periodo 2000-2018.

\section{Resultados}

En el periodo 2000-2018, los sectores que participaron en promedio con un porcentaje significativo en el Producto Interno Bruto (PIB) Nacional, a precios del $2007^{5}$, fueron los siguientes: Manufactura (excepto refinación de petróleo) 11.86, petróleo y minas 10.56 , comercio también con el 10.56, la construcción con el 8.42,

${ }^{4}$ Productos derivados over the counter como los Credit Default Swaps.

${ }^{5}$ El PIB real o a precios constantes se refiere al valor monetario de todos los bienes y servicios producidos al interior del país, valorados según los precios de un determinado año que se toma como referencia para realizar comparaciones (en el caso ecuatoriano corresponde al año 2007), para eliminar la variación de los precios a lo largo del tiempo, indicando realmente las variaciones de la economía. enseñanza y servicios sociales y de salud con 8.18, y agricultura con el $7.93 \%$ (Ver Figura 1). La construcción se ubicó entre los seis de los dieciocho sectores económicos ${ }^{6}$ que más aportaron al PIB Nacional en términos reales, situándose en el tercer lugar con el $8.42 \%$, después de los sectores petróleo y minas y comercio que ocuparon el segundo lugar con un $10.56 \%$ cada uno.

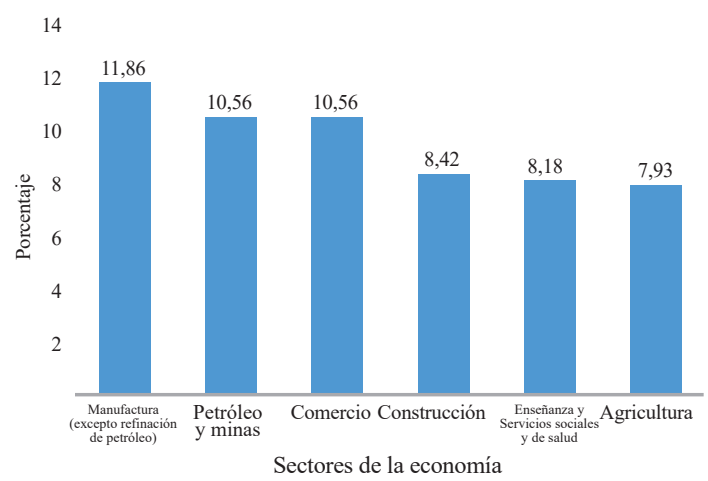

Figura 1. Sectores económicos con mayor participación promedio en el PIB en el periodo 2000 - 2018.

Fuente: Banco Central del Ecuador.

El porcentaje de participación de la construcción en el PIB se prevé que muy levemente decrecerá en los próximos tres años. Este comportamiento obedece a la alta correlación $^{7}$ que existe con el comportamiento de la economía en función de la política económica. Sachs y Larraín (1994) indica que la teoría de Jan Tinbergen intenta conceptualizar cómo deben actuar las autoridades económicas, tiene un carácter normativo a través de la política fiscal y de la política monetaria,

\footnotetext{
${ }^{6}$ Agricultura, ganadería, casa y silvicultura; acuicultura y pesca de camarón; pesca (excepto camarón); petróleo y minas; refinación de petróleo; manufactura (excepto refinación de petróleo); suministros de electricidad y agua; construcción; comercio; alojamiento y servicios de comida; transporte; correo y comunicaciones; actividades de servicios financieros; actividades y técnicas y administrativas; enseñanza y servicios sociales y de salud; administración pública, defensa y planes de seguridad social obligatoria; servicio doméstico; otros servicios; $\mathrm{y}$, otros elementos del PIB.

${ }^{7}$ La correlación explica la relación que existe entre las variables dependiente e independiente.
} 
para corregir problemas de liquidez que se reflejan por la desaceleración económica, de tal forma que repercute en la dinámica de la construcción. Kumar (2011) afirma que la expansión de la actividad de la construcción está encabezada por un aumento en la expansión económica. El sector de la construcción incluye: Construcción de proyectos de infraestructura, de edificios, y casas, además la construcción de viviendas para fines residenciales como no residenciales, sin embargo, con la desaceleración económica que comenzó en el 2007-2008, la demanda de trabajo de las empresas de la construcción comenzó a disminuir.

La variación de la construcción en los años 2001 y 2002 fue del 23.31 y $20.96 \%$ respectivamente, decreciendo drásticamente al siguiente año, y la variación del PIB en los mismos años creció del 4.02 al 4.10\%, luego durante los siguientes ocho años tanto la construcción como el PIB registraron una variación positiva, hasta que el primero volvió a crecer significativamente en el $17.55 \%$ en el 2011 y $12.21 \%$ en el 2012, como se observa en la Figura 2. Es importante señalar que a partir del 2008 hasta el año 2014 el crecimiento del sector fue mayor al de la economía nacional, debido a los recursos asignados por el Banco del Instituto de Seguridad Social (BIESS) y por la adquisición de bienes inmuebles como solución a necesidades de vivienda y de oficinas, así como también a la ejecución de vías terrestres ejecutadas por el gobierno de Rafael Correa, quien mantuvo una política de intervención aumentando progresivamente el peso del sector público. Acosta-Burneo (2018), afirma que lamentablemente la inversión pública no genera más riqueza en la sociedad porque las malas decisiones de inversión dilapidan el escaso ahorro nacional y el patrimonio de los ecuatorianos. En todo caso, la inversión social contribuye para que el empresario sea eficiente. A partir del 2013 empezó a decrecer significativamente por los insuficientes recursos económicos en el gobierno, de tal forma que el PIB al año 2016 decreció en $1.58 \%$. Inicialmente se pronosticó que en los años 2017 y 2018 siga decreciendo, situación que no se dio debido al endeudamiento agresivo en el que incurrió el Gobierno ${ }^{8}$, de tal forma que el Banco Central del Ecuador (2018) proyectó que crecería la economía en el $1.1 \%$ en el año 2018. Asimismo la Asamblea Nacional (El Ciudadano, 2017) aprobó la proforma presupuestaria manteniéndose con una proyección de 2.39 para el 2019 y $2.49 \%$ en el 2020 . En ese momento de transición, el mayor rol lo jugó el privado, aquel sector que en el 2006 era responsable del $70 \%$ de la inversión de largo plazo y que cedió espacio para que el Estado en 2014 lo supere llegando al 55\% (Ortiz, 2018).

Según Durán (2017) el Fondo Monetario Internacional (FMI) y la Comisión Económica para América Latina (CEPAL) manifestaron que el PIB crecería en el $0.6 \mathrm{y}$ en el $1.0 \%$ en el 2018 , en su orden, mientras que para el mismo año Artola (2018) estimó que cerraría con un

\footnotetext{
${ }^{8}$ A diciembre de 2017 la deuda externa fue de 31.749 .800 USD incrementándose en el $198.6 \%$ en relación al 2007 , de igual manera en el mismo periodo la deuda interna creció, drásticamente, en el 356\% (Ministerio de Economía y Finanzas, 2017).
} 
crecimiento del $1.8 \%$, sin embargo, debido a la apertura del gobierno, el FMI prevé que el Ecuador crezca un poco más en el 2019 (El Telégrafo, 2018). Se puede afirmar que esta relación de crecimiento, construcción - PIB, históricamente no es proporcional como se observa en la Figura 2.

La Industria de la construcción incentiva el crecimiento de la economía, como se refiere Velasco (2015), que el segmento inmobiliario, se convirtió en un gran dinamizador de la economía, incentivado especialmente por la gran cantidad de recursos asignados por el BIESS para créditos hipotecarios (en el 2014 se colocó 1.261 millones de dólares) y por la participación de inversionistas y compradores que vieron en la adquisición de bienes inmuebles, el mejor atractivo de inversión y de solución a necesidades de vivienda y oficinas.

Luego del impresionante crecimiento del sector de la construcción en el año 2011, es a partir de dicho año donde también se inició una desaceleración progresiva a pesar que el precio del petróleo se mantuvo en casi 100 dólares en promedio por barril entre 2011 y el 2014, es decir en este periodo el país dispuso de mayores recursos económicos. Hurtado (2017) afirmó que cuando se produjo la caída del precio del petróleo, Correa privatizó empresas públicas, se interesó en la inversión extranjera, eliminó subsidios, buscó la cooperación del sector privado y acordó un tratado de libre comercio con la Unión Europea. La desaceleración fue de la mano con el comportamiento de la economía nacional, especialmente en el año 2016 cuando la economía decreció. En los dos años siguientes, pese a que el precio del petróleo bajo sustancialmente, el gasto del gobierno se mantuvo y sumado a la apreciación del dólar dieron como resultado el decrecimiento de la economía. Este comportamiento se debió a la política económica implementada en ese entonces. Al respecto, Dahik (2015) atribuyó al modelo económico y a la presión que ejerce sobre el gasto público la causa de la crisis que atraviesa la economía ecuatoriana. Si bien señaló que la baja del precio del petróleo es el detonante, las causas de la profunda crisis se deben a un un conjunto de medidas como el impuesto del $5 \%$ (a la salida de capitales), la falta de

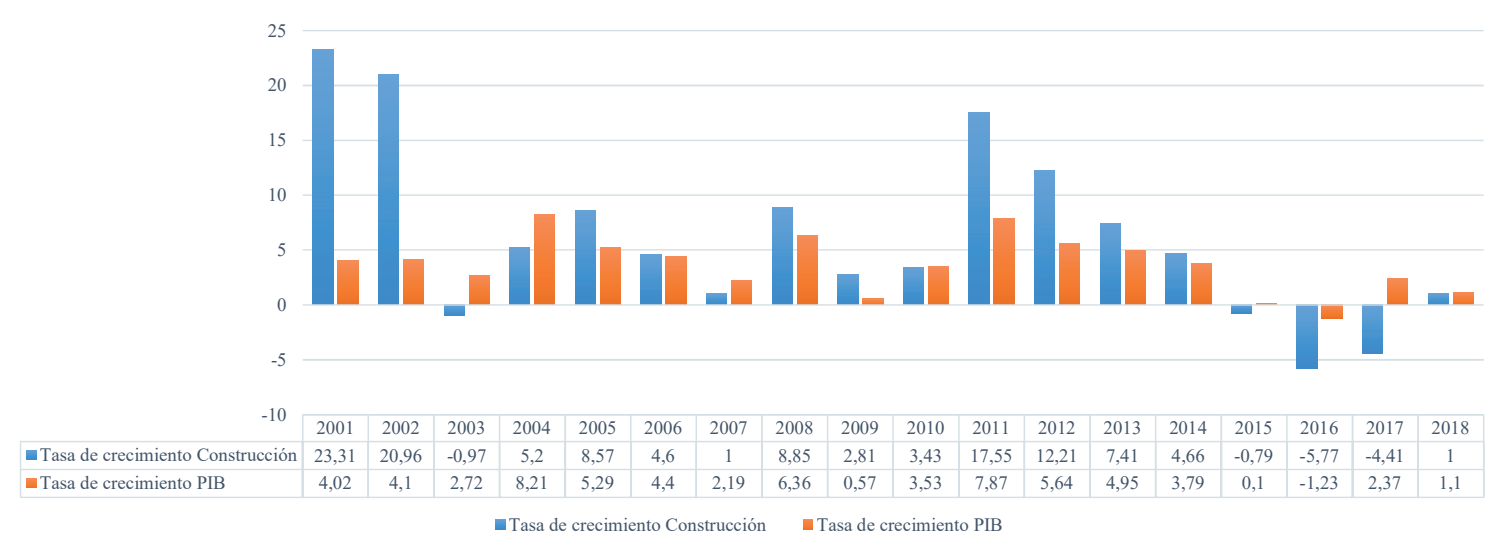

Figura 2. Variación del Sector de la Construcción y del PIB. Fuente: Banco Central del Ecuador. 


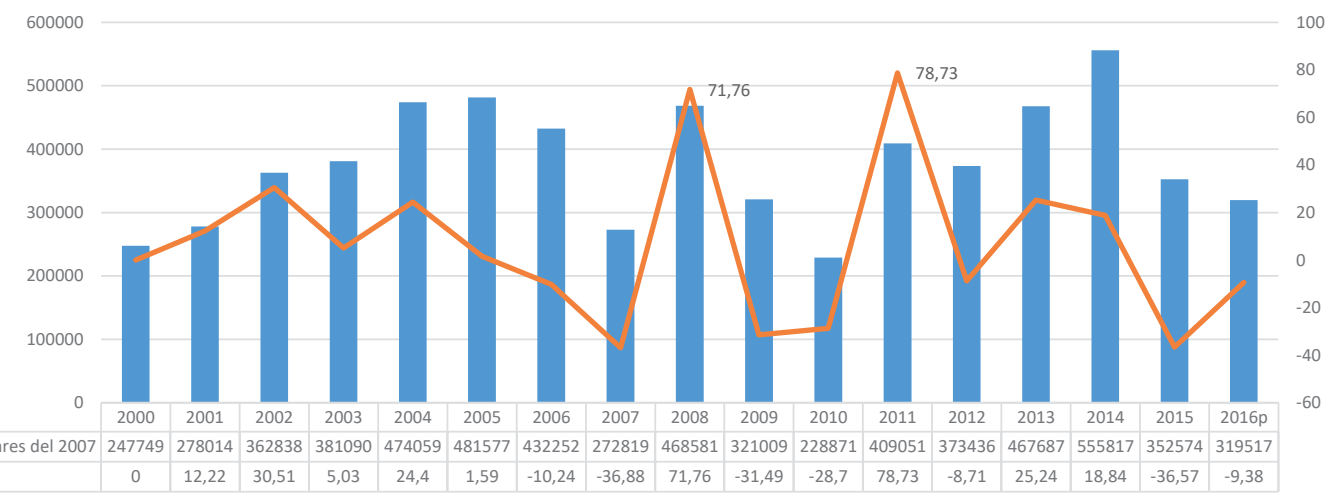

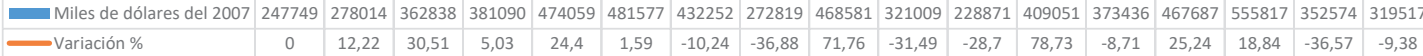

Cuariación \%
Figura 3. Variación de la Formación Bruta de Capital Fijo de la Construcción $2000-2016$.

Fuente: Banco Central del Ecuador.

inversión extranjera, el excesivo gasto público y las salvaguardias.

Como es de conocimiento público, el sector de la construcción en los años 2008 y 2011 crecieron en el $71.76 \%$ y en el $78.73 \%$, respectivamente, sin embargo el año de mayor inversión fue el 2014 (ver Figura 3), impulsada por el Gobierno Nacional en infraestructura de salud y educación, sectores estratégicos, en viabilidad y vivienda de interés social; $y$, por parte del sector privado en la construcción especialmente de vivienda para sectores económicos medios altos y altos, así como también edificaciones para sectores de turismo e industria.

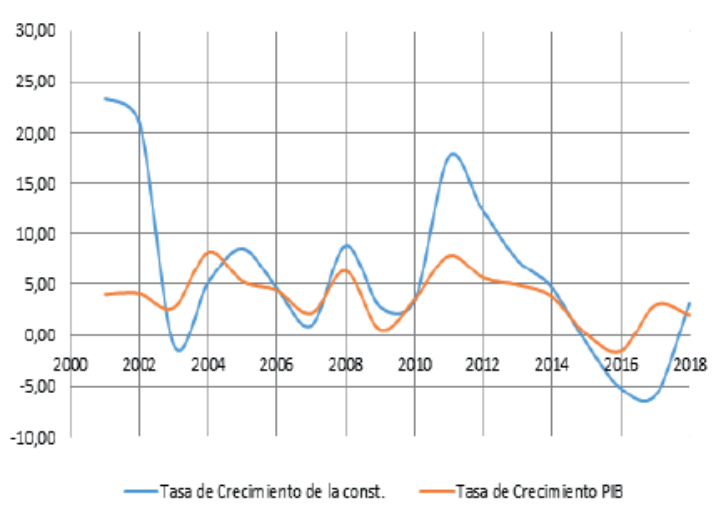

Figura 4. Ciclos económicos del PIB y la Construcción. Fuente: Banco Central del Ecuador.
En la Figura 4 se observan los ciclos económicos siguientes: 2003-2007 (cuatro años), 2007-2010 (tres años), 2010-2016 (6 años) y 2016 posiblemente hasta 2018 (dos años). Es así que Giudice (2004) manifiesta que los ciclos son desviaciones temporales de la economía y que la política fiscal y monetaria son herramientas de política por perfeccionar. Además, demuestra que el ciclo más corto sería el último, por la mala situación económica que atraviesa el país y las expectativas de obtener un mayor flujo de efectivo o de ingresos, de tal forma que la recuperación de la economía no se daría en el corto plazo, sino más bien en el mediano. Oliva (2018) cuestiona si en la actualidad con un nuevo liderazgo en la conducción del Estado, podrá Ecuador, con una política económica heterodoxa ${ }^{9}$, conseguir otra victoria ${ }^{10}$ y evitar el ajuste

\footnotetext{
${ }^{9}$ Es la búsqueda de explicaciones científicas, claras y rigurosas de la dinámica real de la economía. No se trata solo de la construcción de explicaciones alternativas al modelo neoclásico, sino fundamentalmente de aceptar y afrontar el reto cognitivo y explicativo de los fenómenos que enfrentan los economistas (UNAM, 2010).

${ }^{10}$ Ecuador con viento en contra y sin soberanía monetaria ha logrado reflotar la economía en el año 2017 [....]. Ecuador gano la primera batalla al neoliberalismo, que en momentos difíciles aumenta la presión por el ajuste social (Oliva, 2018).
} 
neoliberal en 2018. Se abre una gran interrogante ahora que Correa y su equipo económico han salido del gobierno, pues todavía no se dilucida cual es la visión económica de Lenín Moreno, sin embargo, el Banco Central del Ecuador mantiene un escenario optimista en la recuperación de la economía no así el FMI ni la CEPAL.

Velasco (2015) señala que de la obra pública varios proyectos están concluidos y otros en marcha, cuya ejecución debido a la limitación de liquidez de los organismos estatales, se han visto afectados los contratistas y toda la cadena relacionada a esta actividad, debido a la falta de pago por los trabajos ejecutados, con las consiguientes repercusiones empresariales que esta situación representa. Uno de los efectos más visibles es el empleo, considerando que este sector absorbe mano de obra calificada y no calificada. Pita (2018) comenta que la construcción genera directamente más o menos 500 mil plazas de trabajo e indirectamente cerca de 1,3 millones personas más que se relacionan con la fabricación, distribución y comercialización de los bienes y servicios. En el periodo 2000-2017 la población ocupada en construcción representa el 6.95\%. Sin embargo, los sectores que más emplean mano de obra son: Comercio, agricultura y caza, manufactura (excepto refinación de petróleo), enseñanza y servicios sociales de salud; la construcción se ubica en el quinto lugar (Ver Figura 5).

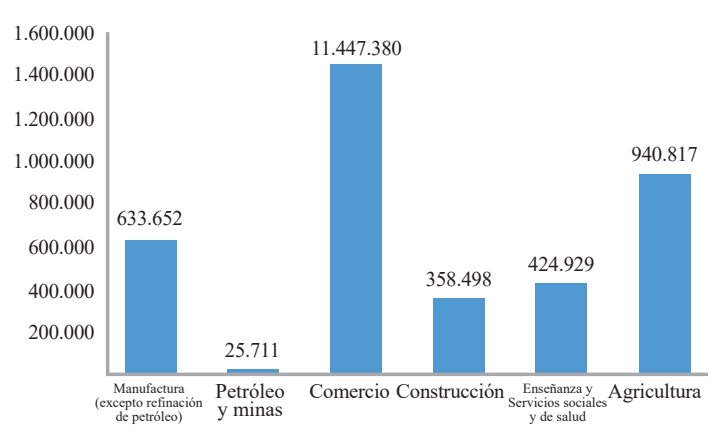

Figura 5. Sectores de la economía con mayor población ocupada $2000-2017$.

Fuente: Encuesta Nacional de Empleo, Desempleo y Subempleo - ENEMDU, 2000-2017 (INEC, 2018).

En la Figura 6 se aprecia la participación y evolución de la población ocupada en el Sector de la Construcción durante el periodo 2000-2017, siendo el año 2013 el de mayor participación y crecimiento, a pesar que el crecimiento de la economía se encontraba en desaceleración a partir del 2011. Este

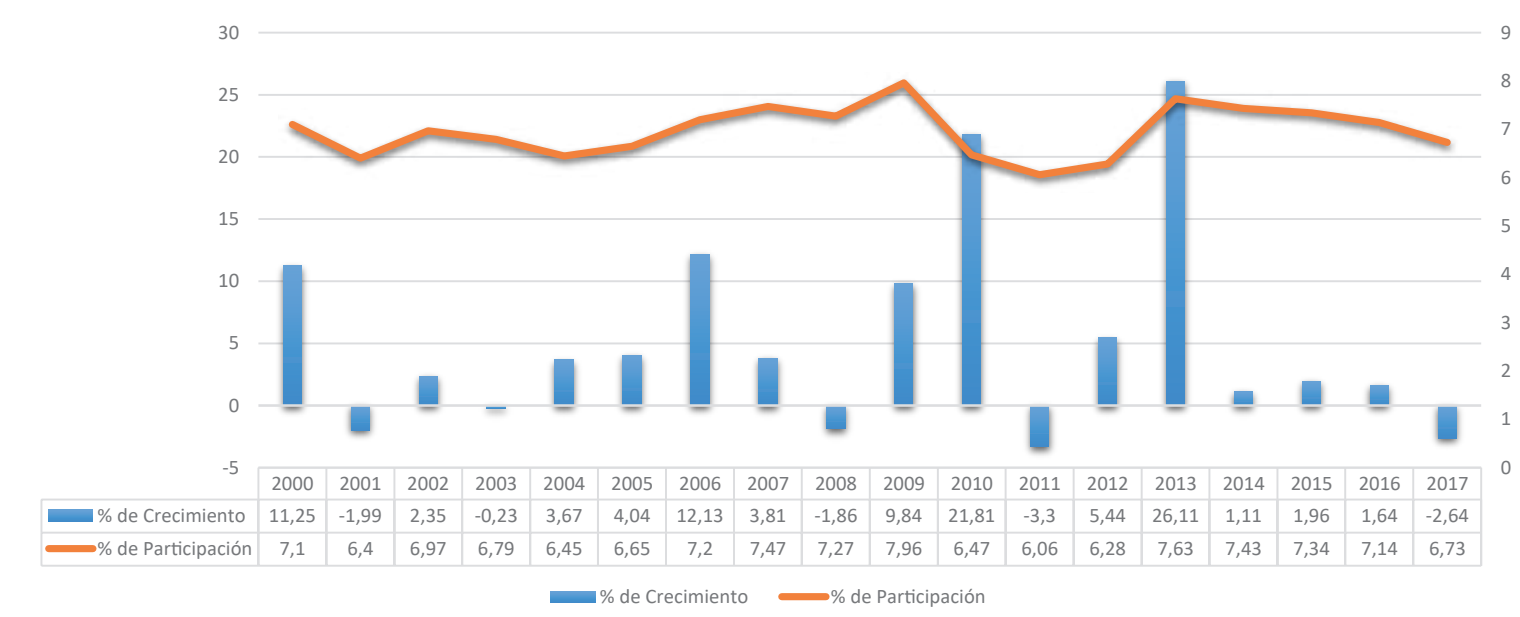

Figura 6. Participación de la población ocupada en el Sector de la Construcción y su crecimiento. Fuente: Encuesta Nacional de Empleo, Desempleo y Subempleo - ENEMDU, 2000-2017 (INEC, 2018).

\section{4}

PODIUM No. 35, Junio 2019, pp. 57-68

Universidad Espíritu Santo - UEES

ISSN: 1390-5473 e-ISSN: 2588-0969 
fenómeno obedece al ciclo más largo, 6 años, del periodo de estudio. En este ciclo económico la tasa de crecimiento de la población ocupada de dicho sector cayó drásticamente al $1.11 \%$ al año 2014 debido al decaimiento de la economía por las razones antes mencionadas. Este fenómeno de la población ocupada es muy sensible al comportamiento general de la economía.

\section{Conclusiones}

La industria de la Construcción es uno de los sectores que más influyen en el comportamiento del PIB. El stock de acciones de capital dinamizó la economía nacional, es así que el crecimiento del 2011 con respecto al 2010 se ubicó en el $78.73 \%$. En el 2014 se registró la mayor cantidad de inversión (555 817 miles de dólares del 2007) desde el año 2000, sin embargo si relacionamos la variación del sector en ese año se observó un crecimiento del $4.66 \%$ inferior al registrado en el 2013 que se ubicó en el $7.41 \%$ a pesar de que en éste año la inversión fue menor, es decir el comportamiento de la industria de la construcción no es proporcional a la inversión pública y privada. La política aplicada por Rafael Correa tuvo implicaciones en el sector real de la economía, no sólo porque se envió a la Asamblea Nacional una ley que aumentaba en forma sustancial el impuesto a la plusvalía, al gravar hasta el $75 \%$ del beneficio obtenido por la venta de bienes raíces (Hurtado, 2017), sino también por un proceso de desinversión pública (privatizaciones). La tasa de crecimiento de la construcción superó a la del PIB en los años 2011 y 2012, así como también en el sector real existió variaciones significativas.

De los cuatro ciclos económicos, el más largo fue el del periodo 2010-2016 llegando al año pico en el 2011, año en el cual se verificó una mayor inversión de parte del Estado Ecuatoriano, situación que obedeció a que el precio promedio del petróleo en ese año superó los 100 dólares por barril, pero a partir del 2015 empezó a bajar drásticamente, y con un modelo de excesivo gasto público, causa principal, se dio la crisis económica en el año 2016.

Se estima que en los próximos años se concrete las alianzas público-privado que permitirán mejorar la economía ecuatoriana mediante el incremento de la inversión interna para lo cual se requiere estabilidad fiscal y jurídica, de tal forma que en el futuro sería bueno realizar una investigación sobre el cambio de modelo económico en el Ecuador.

\section{Referencias}

Barranquero, A., y Sáez, B., Ch. (2012). Teoría Acosta-Burneo, A. (30 de Noviembre de 2018). ¿Más inversión pública? Revista Vistazo. Recuperado de: https://www.vistazo.com/seccion/mas-in version-publica.

Ahmadi, N., y Shahandashti, M. (2017). Comparative empirical analysis of temporal relationships between construction investment and economic growth in the United States. Construction Economics and Building, 17(3), 85-108. doi:10.5130/AJCEB.v17i3.5482

Artola, V. (15 de Octubre de 2018). Ecuador prevé un crecimiento de hasta $1.2 \%$ en 2018 . Revista Vistazo. Recuperado de: 
https://www.vistazo.com/seccion/pais/po litica-nacional/ecuador-preve-un-crecimi ento-de-hasta-12-en-2018

Banco Central del Ecuador. (2018). Información Estadística Mensual Marzo-Abril 2018. Recuperado de:

https://contenido.bce.fin.ec/home1/estadi sticas/bolmensual/IEMensual.jsp .

Banco Central del Ecuador. (2018). Noventa años del Banco Central del Ecuador. Series estadísticas históricas 1927-2017. Recuperado de:

https://www.academia.edu/36213398/Ba nco_Central_del_Ecuador_SUBGEREN CIA_DE_PROGRAMACI $\%$ C $3 \% 93 \mathrm{~N}$-Y REGULACI\%C3\%93N_DIRECCI\%C3 $\% 93$ N_NACIONAL_DE_S\%C3\%8DNT ESIS_MACROECON $\%$ C $3 \% 93 \mathrm{MICA}$

Banco Central del Ecuador (2018) Indicadores económicos. Recuperado de: https://contenido.bce.fin.ec/documentos/P ublicacionesNotas/Catalogo/CuentasNaci onales/Anuales/Dolares/indiceFBKF.htm

Daher. A. (2013). El sector inmobiliario y las crisis económicas. EURE Santiago 39(118), 47-76. https://dx.doi.org/10.4067/S0250-716120 13000300003

Dahik, A. (26 de Octubre de 2015). No es el petróleo la causa de la crisis, es el gasto público. Ecuadorenvivo.com. Recuperado de: http://www.ecuadorenvivo.com/economi a/85-sp-651/37864-no-es-el-petroleo-el-c ausante-de-la-crisis-es-el-gasto-publico-a lberto-dahik-exvicepresidente-dela-repub lica.html\#.W4_2a3pzuCg.

Durán, S. (27 de Septiembre de 2017). La Construcción en Ecuador. Construcción Latinoamericana $C L A$. Recuperado de:

https://www.construccionlatinoamerican a.com/la-construccion-en-ecuador/12951 0. article

Durán. S. (2017). Oportunidades del sector de la Construcción Ecuatoriano para el 2018.
En IV Foro Agenda Empresarial 2018. Conferencia llevada a cabo en UDLA, Quito-Ecuador.

El Ciudadano. (9 de Enero de 2017). Asamblea aprobó proforma presupuestaria y programación cuatrianual. Ecuadorinmediato.com. Recuperado de: http://www.ecuadorinmediato.com/index. php module $=$ Noticias $\&$ func $=$ news user _view\&id=2818825176

El Telégrafo. (18 de Abril de 2018). FMI predice crecimiento para Ecuador y la región. $E l$ Telégrafo. Recuperado de:

https://www.eltelegrafo.com.ec/noticias/eco nomia/4/fmi-crecimiento-ecuador-economia

Estefanía, J. (2016). Una lectura de Las posibilidades económicas de nuestros nietos de John M. Keynes. Madrid: Taurus.

Giudice, V. (2004). La Escuela Neokeynesiana: Desarrollo y aportes. Pensamiento Crítico 3, 25-31.

Gowan, P. (2009). Crisis en el Corazón del Sistema. Consecuencia del Nuevo Sistema de Wall Street. Madrid, España: Akal.

Hurtado, O. (2017). Ecuador entre dos siglos. Penguin Random House Grupo Editorial.

INEC. (2018). Encuesta Nacional de Empleo, Desempleo y Subempleo - ENEMDU, 2000-2017. Recuperado de: www.ecuadorencifras.gob.ec

Kumar, A. (2011). A causal analysis between construction flows and economic growth: evidence from India. Journal of International Business and Economy, 12(2), 27-42.

Ministerio de Economía y Finanzas. (2017). Deuda Pública del Sector Público Ecuatoriano. Recuperado de: https://www.finanzas.gob.ec/wp-content/ uploads/downloads/2018/01/DEUDA-SE CTOR-P\%C3\%9ABLICO-DEL-ECUA 
DOR_diciembre2017-1.pdf.

Oliva, N. (2018). Ecuador 2018: ¿Quién pagará por el ajuste?, [mensaje en un blog]. Blog Observatorio de la Dolarización. Recuperado de:

https://dolarizacionec.wordpress.com/20 18/01/29/ecuador-2018-quien-pagara-elajuste-por-nicolas-oliva/

Ortiz, F. (28 de Diciembre de 2018). 2019: Año de la transición. Revista Vistazo. Recuperado de: https://www.vistazo.com/seccion/pais/actu alidad-nacional/2019-ano-de-la-transicion

Pita, E. (2018). El sector de la construcción representa un $10 \%$ del PIB, [mensaje en un blog]. Blog Vive1.com. Recuperado de: http://blog.vive1.com/el-sector-de-la-const rucci $\%$ C3\%B3n-representa-un-10-del-pib

Radziunas, J. (2005). Una visión poskeynesiana del gasto público. Revista Equidad y Desarrollo, 1(5), 81-94.

https://doi.org/10.19052/ed.358

Rincón, I. y Torres, O. (2013). Epistemología económica del pensamiento neokeynesiano. Contribuciones a la Economía, Julio 2013. Recuperado de: www.eumed.net/ce/2013/keynesianismo. html.

Sachs, J. y Larraín, F. (1994). Macroeconomía en la economía global. México D.F.: Prentice Hall Hispanoamericana.

UNAM. (2010). ¿Qué es la economía heterodoxa? Seminario de Microeconomía Heterodoxa. Recuperado de:

http://www.economia.unam.mx/smh/eco nomia-heterodoxa.html

Velasco, M.I. (1015). La Construcción es sensible a ciclos económicos [mensaje en un blog]. Blog Vive1.com. Recuperado de: http://blog.vive1.com/el-sector-de-la-con strucci $\% \mathrm{C} 3 \% \mathrm{~B} 3 \mathrm{n}$-es-el-m $\% \mathrm{C} 3 \% \mathrm{~A} 1 \mathrm{~s}-\mathrm{sen}$ sible-a-ciclos-econ $\% \mathrm{C} 3 \% \mathrm{~B} 3$ micos 
